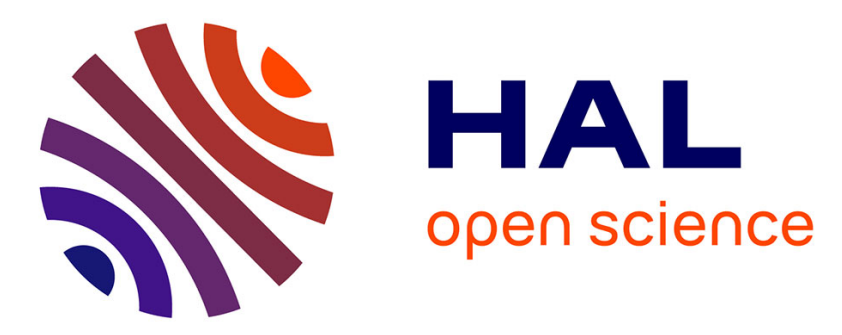

\title{
Characterisation of the Tri-Modal Discrete Sea Clutter Model
}

Luke Rosenberg, Sébastien Angelliaume

\section{To cite this version:}

Luke Rosenberg, Sébastien Angelliaume. Characterisation of the Tri-Modal Discrete Sea Clutter Model. RADAR2018, Aug 2018, BRISBANE, Australia. hal-02183176

\section{HAL Id: hal-02183176 https://hal.science/hal-02183176}

Submitted on 15 Jul 2019

HAL is a multi-disciplinary open access archive for the deposit and dissemination of scientific research documents, whether they are published or not. The documents may come from teaching and research institutions in France or abroad, or from public or private research centers.
L'archive ouverte pluridisciplinaire HAL, est destinée au dépôt et à la diffusion de documents scientifiques de niveau recherche, publiés ou non, émanant des établissements d'enseignement et de recherche français ou étrangers, des laboratoires publics ou privés. 


\title{
Characterisation of the Tri-Modal Discrete Sea Clutter Model
}

\author{
Luke Rosenberg* and Sébastien Angelliaume $^{\dagger}$ \\ ${ }^{*}$ Defence Science and Technology Group, Australia, ${ }^{\dagger}$ ONERA, France \\ Email: Luke.Rosenberg@dst.defence.gov.au
}

\begin{abstract}
Accurately representing the sea clutter amplitude or intensity distribution is important for achieving a constant false alarm rate in a detection scheme. This can be difficult as the backscatter statistics change with the sea surface characteristics, the geometry of acquisition and the radar parameters. Recently, a new compound distribution model has been proposed which models the sea clutter texture discretely, with each component representing different scattering components. In this paper, the tri-modal discrete texture (3MD) model is explored using real aperture data from the Defence Science and Technology Group Ingara radar and synthetic aperture radar data from the French Aerospace Laboratory (ONERA) SETHI radar. Both the model fitting accuracy and the variation of the texture components are studied to better understand how to relate the model to the underlying sea-clutter characteristics.
\end{abstract}

\section{INTRODUCTION}

The study and analysis of sea clutter is important in many different applications such as oceanography, maritime surveillance and target classification. The problem of detecting ships in either real or synthetic aperture radar (SAR) data is typically approached with constant false alarm rate (CFAR) detection algorithms using a probability density function (PDF) model to characterise the target free data. Models for the amplitude distribution of sea clutter are usually developed empirically from measurements of real data as it is not currently possible to accurately predict the PDF of sea clutter under different conditions using physical models of the sea surface.

There has been a long development of PDF models used to fit both real aperture radar and synthetic aperture radar. With coarse range resolution, a reasonable model for the seaclutter PDF is the Gaussian distribution, with the envelope of the returns given by a Rayleigh distribution. As the range resolution becomes finer, the variation of the sea swell is better resolved and the effect of breaking waves and other discrete events (sea-spikes) are more pronounced. These returns have a larger magnitude which has led to the development of newer PDF models with longer 'tails' such as the log-normal and Weibull distributions. A popular and widely used framework for developing PDF models is the compound Gaussian model which was originally proposed for use in sea-clutter by Ward [1]. This model includes a temporal or fast varying component known as speckle which relates to the Bragg scattering, and a slowly varying component which captures the underlying swell and models the texture.
Compound distribution models include the $\mathrm{K}$ or $\mathrm{K}+$ noise [2], KA [2], KK [3], Pareto or Pareto+noise [4]-[7] and the $\mathrm{K}+$ Rayleigh [8]. While the KA and KK distributions model the sea clutter very accurately, they are difficult to implement in practice. Consequently, the Pareto model has become popular due to its smaller number of parameters and ability to account for thermal noise. The $\mathrm{K}+$ noise distribution has also been extended to a $\mathrm{K}+$ Rayleigh [8] distribution by taking into account any extra Rayleigh scattering not captured by the thermal noise. This model has been shown to fit both real and synthetic aperture radar data very accurately and over a wide range of geometries [9]. Note that many of these are only first order compound Gaussian models as they cannot model the speckle correlation independently of the thermal noise.

The tri-modal discrete texture (3MD) model [10], [11] is another candidate which has demonstrated great potential for modelling synthetic aperture radar (SAR) sea-clutter and is unique in the way it models the sea clutter texture as a combination of discrete components. The two main contributions in this paper are to first investigate the suitability of this model on two different airborne datasets and to study the distribution modes to determine their relationship to the characteristics of the underlying sea-clutter. The two data sets include the Ingara X-band medium grazing angle data set collected by the Defence Science and Technology (DST) Group in Australia and both L-band and X-band synthetic aperture radar (SAR) data collected by the SETHI radar of the French Aerospace Laboratory (ONERA). This contrast is important as the process of SAR image formation alters the radar backscatter with the SAR representation of ocean waves being different from that of real aperture radar [9].

In Section II, a number of key PDF models are described with details on how their parameters are estimated and the model fits assessed. The two datasets are then presented in Section III with a study of the model fits and modes used for the 3MD model. Section IV then looks further at the texture estimates of the $3 \mathrm{MD}$ model over a wide parameter space.

\section{AMPLITUDE DISTRIBUTION MODELS}

To understand the development of the compound distribution, consider a radar receiving in-phase and quadrature data from an external clutter source with its amplitude defined by Gaussian statistics with zero mean and variance, $x$. In addition, thermal noise from the radar will add a component $\sigma_{\mathrm{n}}^{2}$ which is included by offsetting the variance $x$. In target 
detection analysis, the envelope of the received pulses is often converted to power (square law) and the clutter distribution becomes exponential. This component is known as speckle in the compound representation. For a frequency agile or scanning radar with sufficient time between looks, a common method to improve the detection performance is to sum a number of looks. If there are $M$ independent exponential random variables, $z=\sum_{m=1}^{M} y_{m}$, then the received power is described by a gamma PDF,

$$
P(z \mid x)=\frac{z^{M-1}}{\left(x+\sigma_{\mathrm{n}}^{2}\right)^{M} \Gamma(M)} \exp \left[-\frac{z}{x+\sigma_{\mathrm{n}}^{2}}\right]
$$

where $0 \leq x \leq \infty$. To include the texture component which modulates the speckle, we integrate over the speckle mean power,

$$
P(z)=\int_{0}^{\infty} P(z \mid x) P(x) d x
$$

where $P(x)$ is the distribution of the texture component. While there are analytic solutions in many cases, when noise is included in the model, numerical integration must be used to evaluate the compound distribution.

\section{A. K+Rayleigh model}

The most commonly used PDF model for sea-clutter in both real and synthetic aperture radar is the K-distribution, or $\mathrm{K}+$ noise when thermal noise is included. However, in many cases the K-distribution is not able to capture the long tails present due to high magnitude spiky sea clutter returns. The $\mathrm{K}+$ Rayleigh distribution was formalised in [8] and includes a further Rayleigh component to better capture these returns. It is defined by explicitly separating the speckle mean into two components, $x=x_{\mathrm{r}}+\sigma_{\mathrm{r}}^{2}$, where the extra Rayleigh component, $\sigma_{\mathrm{r}}^{2}$ is modelled in the same fashion as the thermal noise. The $\mathrm{K}+$ Rayleigh model uses a gamma distribution for the texture,

$$
P\left(x_{\mathrm{r}} \mid \nu_{\mathrm{r}}, b_{\mathrm{r}}\right)=\frac{b_{\mathrm{r}}^{\nu_{\mathrm{r}}}}{\Gamma\left(\nu_{\mathrm{r}}\right)} x_{\mathrm{r}}^{\nu_{\mathrm{r}}-1} \exp \left[-b_{\mathrm{r}} x_{\mathrm{r}}\right], \quad 0 \leq x_{\mathrm{r}} \leq \infty
$$

where $\nu_{\mathrm{r}}$ is the shape and $b_{\mathrm{r}}=\nu_{\mathrm{r}} / \sigma_{\mathrm{c}}^{2}$ is the scale with the mean clutter power, $\sigma_{\mathrm{c}}^{2}$. The influence of the extra Rayleigh component can be measured by the ratio of the mean of the Rayleigh component, $\sigma_{\mathrm{r}}^{2}$ to the mean of the gamma distributed component of the clutter and is defined by $k_{\mathrm{r}}=\sigma_{\mathrm{r}}^{2} / \sigma_{\mathrm{c}}^{2}$. For the Ingara data, it has typical values in the range $0 \leq k_{\mathrm{r}} \leq 4$. To calculate the compound distribution in (2), the integration is then performed with the modified speckle mean level, $x_{\mathrm{r}}$ instead of the total speckle $x$. To estimate the distribution parameters, there are a number of techniques such as the method of moments, the $\langle z \log z\rangle$ method or a least squares minimisation between the data and model complementary cumulative distribution functions (CCDF) [12].

\section{B. Pareto model}

The Pareto model is described by only two parameters (shape and scale), yet can reasonably model the long tails present in sea-clutter distributions. It was first used for seaclutter modelling by Balleri et al. [4] and later by others at
US Naval Research Laboratory (NRL) and DST Group [5]-[7]. For the Pareto distribution, the texture has an inverse gamma distribution

$$
P(x)=\frac{c^{a}}{\Gamma(a)} x^{-a-1} \exp [-c / x], \quad a>1, c>0
$$

where $a$ is the shape and $c=\sigma_{\mathrm{c}}^{2}(a-1)$ is the scale. Similarly to the $\mathrm{K}+$ Rayleigh model, the distribution parameters can be estimated using method of moments, the $\langle z \log z\rangle$ method or least squares minimisation.

\section{3MD model}

The compound models in the literature all assume a continuous texture distribution function which suggests a small probability of infinite texture values. This is not physically sound as it cannot be measured by any real radar system. The 3MD model [10], [11] instead proposes the use of a discrete texture model that assumes the sea clutter consists of a finite number of distinct modes or scatterer types, $I$. This implies that the scatterers in the observed scene are realisations from homogeneous clutter random variables with different texture values. In the original work, it was found that $I=3$ modes were sufficient to model distributions from the spaceborne SAR imagery, hence the tri-modal in the name. One of the consequences of this discretisation is that spatial and longtime correlation cannot be modelled as part of the texture, and hence the model is less suitable for clutter simulation. The texture PDF is given by

$$
P(x)=\sum_{n=1}^{I} c_{n} \delta\left(x-a_{n}\right), \quad \sum_{n=1}^{I} c_{n}=1, \quad a_{n}, c_{n}>0
$$

where $\delta(\cdot)$ denotes the delta-function, $\mathbf{a}=\left[a_{1}, \ldots, a_{I}\right]$ are the discrete texture intensity levels and $\mathbf{c}=\left[c_{1}, \ldots, c_{I}\right]$ are the corresponding weightings. The continuous distribution is then given by

$$
P(z)=\frac{M^{M}}{\Gamma(M)} z^{M-1} \sum_{n=1}^{I} c_{n} \frac{\exp \left(-\frac{M z}{\rho_{\mathrm{c}} a_{n}^{2}+\rho_{\mathrm{n}}}\right)}{\left(\rho_{\mathrm{c}} a_{n}^{2}+\rho_{\mathrm{n}}\right)^{M}}
$$

with $\rho_{\mathrm{c}}+\rho_{\mathrm{n}}=\frac{\sigma_{\mathrm{c}}^{2}}{\sigma_{\mathrm{c}}^{2}+\sigma_{\mathrm{n}}^{2}}+\frac{\sigma_{\mathrm{n}}^{2}}{\sigma_{\mathrm{c}}^{2}+\sigma_{\mathrm{n}}^{2}}=1$.

\section{Error metrics}

To evaluate how well a model fits a set of observations, there are many statistical tests and measurement techniques in the literature including the mean squared error of the distribution model compared to the data [7], the chi-square and Kolmogorov-Smirnov tests and the Bhattacharyya distance [13]. The first metric used in this paper is the Bhattacharyya distance (BD) which captures the similarity between the actual PDF, $P(\cdot)$ and the theoretical distribution, $Q(\cdot)$

$$
D_{\mathrm{BD}}=-\ln \left(\sum_{x_{k}} \sqrt{P\left(x_{k}\right) Q\left(x_{k}\right)}\right)
$$


where $x_{k}$ represents the data samples. The $\mathrm{BD}$ ranges from 0 to $\infty$, where equal distributions have a distance measure of 0 . To have more readable results, the $\mathrm{BD}$ is reported in $\mathrm{dBs}$. The second metric is the threshold error which is determined by first calculating the CCDF for both the empirical data and the data fit. The threshold error is then the absolute difference between the two results at a fixed CCDF value. This view of the data is important due to its relationship with the threshold in a detection scheme used for distinguishing between targets and interference. In this context, it is commonly referred to as the probability of false alarm.

\section{DATA SELECTION}

\section{A. Ingara real beam data}

Ingara is a polarimetric radar system maintained and operated within the DST Group in Australia [14]. During the ocean backscatter collections in 2004 and 2006, it was operated at $\mathrm{X}$-band in a circular spotlight-mode where the aircraft flew a circular orbit in an anti-clockwise direction (as seen from above) around a nominated point of interest. Each day the radar platform performed at least six full orbits around the same patch of ocean to cover a large portion of grazing angles between $15^{\circ}$ and $45^{\circ}$. The Ingara data has a $200 \mathrm{MHz}$ bandwidth ( $0.75 \mathrm{~m}$ range resolution) and an azimuth resolution of approximately $63 \mathrm{~m}$. Alternate pulses transmitted horizontal $(\mathrm{H})$ and vertical $(\mathrm{V})$ polarisations resulting in a nominal PRF of $300 \mathrm{~Hz}$. For the analysis in this paper, data from a single 2004 flight has been chosen with a Douglas sea state 5 (wind speed of $10.3 \mathrm{~m} / \mathrm{s}$ and a wave height of $2.6 \mathrm{~m}$ ). The data has been pooled into blocks of $5^{\circ}$ azimuth and $3^{\circ}$ grazing with each block containing approximately $10^{6}$ samples. An example of the data is shown in Fig. 1 for the downwind direction and $30^{\circ}$ grazing.

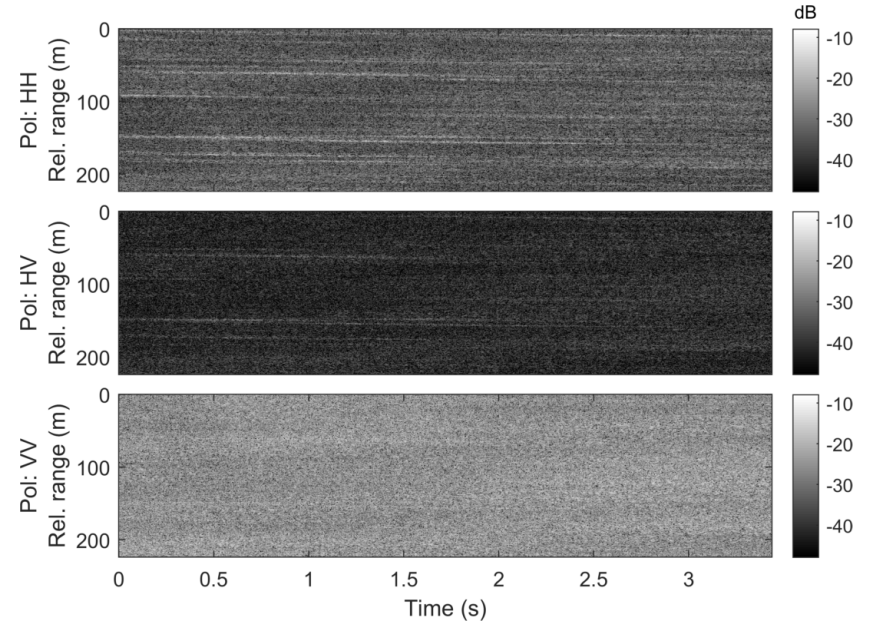

Fig. 1. Ingara data in the downwind direction and $30^{\circ}$ grazing.

Fits of the three models from Section II are then shown in Fig. 2. For each model, the parameters are estimated by a least squares fit to the data CCDF in the log domain. For the 3MD model, the fitting process first assumes a single mode $(I=1)$ and the threshold error is compared with a threshold value of
$0.5 \mathrm{~dB}$. If the error is greater than this threshold, the parameters for a bi-modal fit $(I=2)$ are then estimated. Only if this error is greater than the threshold value, are the parameters estimated for the full 3MD model $(I=3)$. The model components, $c_{n}$, are then ordered from largest to smallest and any modes where the weightings, $a_{n}<10^{-3}$ are removed. Table I shows the estimated parameters of the three models. In this example, both the $\mathrm{HH}$ and $\mathrm{HV}$ polarisations require 3 modes, while VV only requires 2 . The associated error metrics are then reported in Table II where the threshold error is determined at a CCDF value of $10^{-4}$. The $\mathrm{BD}$ results focus on the overall fit and show that the K+Rayleigh model is slightly worse than the Pareto+noise and 3MD models. However, the threshold errors which focus on the distribution tail, reveal that the $\mathrm{K}+$ Rayleigh and Pareto+noise models have a similar fitting error, while the $3 \mathrm{MD}$ model is significantly lower.
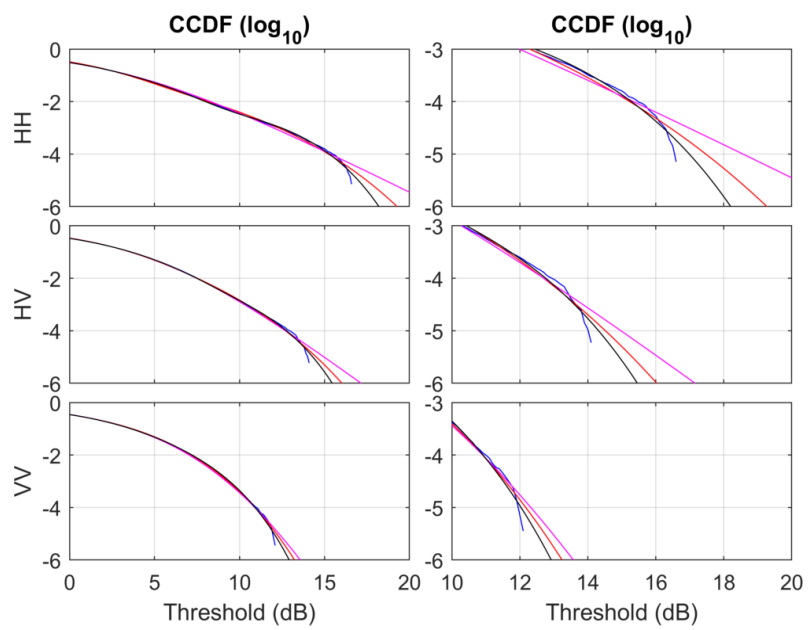

Fig. 2. Ingara CCDF model fits for downwind data and $30^{\circ}$ grazing. Blue data, red - K+Rayleigh, magenta - Pareto+noise, black - 3MD. Right column is a zoomed version of the left.

TABLE I. INGARA PARAMETER ESTIMATES FOR EXAMPLE IN FIG. 2.

\begin{tabular}{|c|c|c|c|}
\hline & HH & HV & VV \\
\hline CNR (dB) & 11.89 & 6.51 & 21.29 \\
K+Rayleigh shape & 0.10 & 0.35 & 8.57 \\
K+Rayleigh $k_{\mathrm{r}}$-value & 0.70 & 0.54 & 0 \\
Pareto shape & 3.2 & 4.83 & 12.58 \\
3MD mode 1 $(a, c)$ & $(0.64,0.76)$ & $(0.51,0.74)$ & $(0.75,0.90)$ \\
3MD mode 2 $(a, c)$ & $(0.35,1.24)$ & $(0.47,1.16)$ & $(0.25,1.27)$ \\
3MD mode 3 $(a, c)$ & $(0.012,2.74)$ & $(0.021,2.04)$ & - \\
\hline
\end{tabular}

TABLE II. INGARA MEASUREMENT ERRORS FOR EXAMPLE IN FIG. 2. THRESHOLD ERROR IS MEASURED AT $10^{-4}$.

\begin{tabular}{|c|c|c|c|}
\hline & HH & HV & VV \\
\hline K+Rayleigh BM (dB) & -30.83 & -34.38 & -36.21 \\
Pareto BM (dB) & -34.97 & -36.67 & -36.88 \\
3MD BM (dB) & -35.94 & -36.02 & -36.26 \\
\hline K+Rayleigh threshold error & 0.25 & 0.14 & 0.20 \\
Pareto threshold error & 0.24 & 0.24 & 0.18 \\
3MD threshold error & 0.095 & 0.096 & 0.086 \\
\hline
\end{tabular}

\section{B. SETHI Synthetic Aperture Radar data}

SETHI is an airborne remote sensing laboratory developed by ONERA [15] and operates as a pod-based system on a 
Falcon 20 Dassault aircraft. In 2015, fully polarimetric SAR data was acquired off the French coast at both $\mathrm{X}$ - and Lbands simultaneously. The SAR imagery has range resolutions of $0.5 \mathrm{~m}$ and $1.0 \mathrm{~m}$ for the $\mathrm{X}$ and L-bands respectively, and the imaged area is processed with an azimuth (alongtrack) resolution equal to the range resolution. The aircraft flew at $2743 \mathrm{~m}(9,000 \mathrm{ft})$ with an imaged area of $8.8 \mathrm{~km}$ in azimuth and $1.1 \mathrm{~km}$ in slant range, covering grazing angles from $38^{\circ}-56^{\circ}$. For this paper, the employed SAR data have been collected over the sea surface in the upwind direction with a wind speed of $10.8 \mathrm{~m} / \mathrm{s}$ (sea state 5-6). Example imagery is shown in Figs. 3 and 4 for the X-and L-band radar systems.

The same PDFs as for the Ingara dataset are now investigated in Fig. 5 for the dual-frequency SAR data. The $\mathrm{K}+$ Rayleigh, Pareto+noise and 3MD models have been fitted to the data with the model parameters shown in Tables III and IV for the X-band and L-band data sets. These results are similar to the Ingara data with a poor match for the Pareto+noise, while the $\mathrm{K}+$ Rayleigh and $3 \mathrm{MD}$ provide better fits. The BD and threshold errors are then given in Tables V and VI. Overall, we find a very good correspondence with the BD being lower than $-33 \mathrm{~dB}$ for each result, implying a consistently good fit to the distribution body. When focusing on the tail of the distribution, low threshold errors are observed for both the $\mathrm{K}+$ Rayleigh and 3MD distributions, while the Pareto+noise model has a greater mismatch.
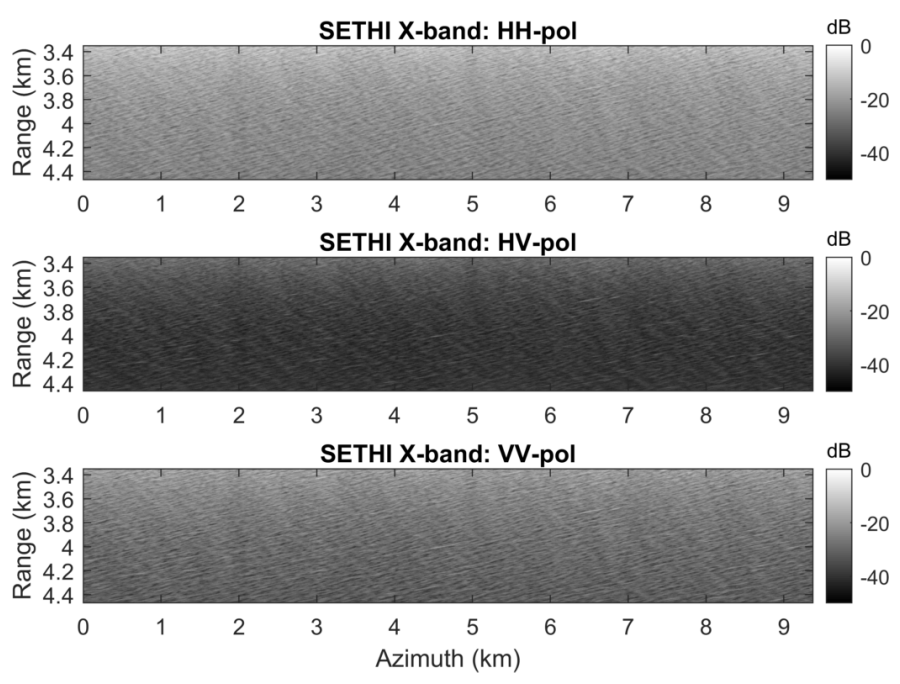

Fig. 3. SETHI X-band SAR data in the upwind direction.

TABLE III. SETHI X-BAND PARAMETER ESTIMATES FOR EXAMPLES IN FIG. 5.

\begin{tabular}{|c|c|c|c|}
\hline & HH & HV & VV \\
\hline CNR (dB) & 33.66 & 28.45 & 36.90 \\
K+Rayleigh shape & 0.76 & 0.43 & 2.73 \\
K+Rayleigh $k_{\mathrm{r}}$-value & 0.29 & 0.53 & 0.04 \\
Pareto shape & 2.95 & 3.91 & 4.03 \\
3MD mode 1 $(a, c)$ & $(0.51,1.17)$ & $(0.49,0.72)$ & $(0.61,1.04)$ \\
3MD mode 2 $(a, c)$ & $(0.46,0.63)$ & $(0.48,1.15)$ & $(0.30,0.61)$ \\
3MD mode 3 $(a, c)$ & $(0.018,2.35)$ & $(0.02,2.23)$ & $(0.088,1.59)$ \\
\hline
\end{tabular}
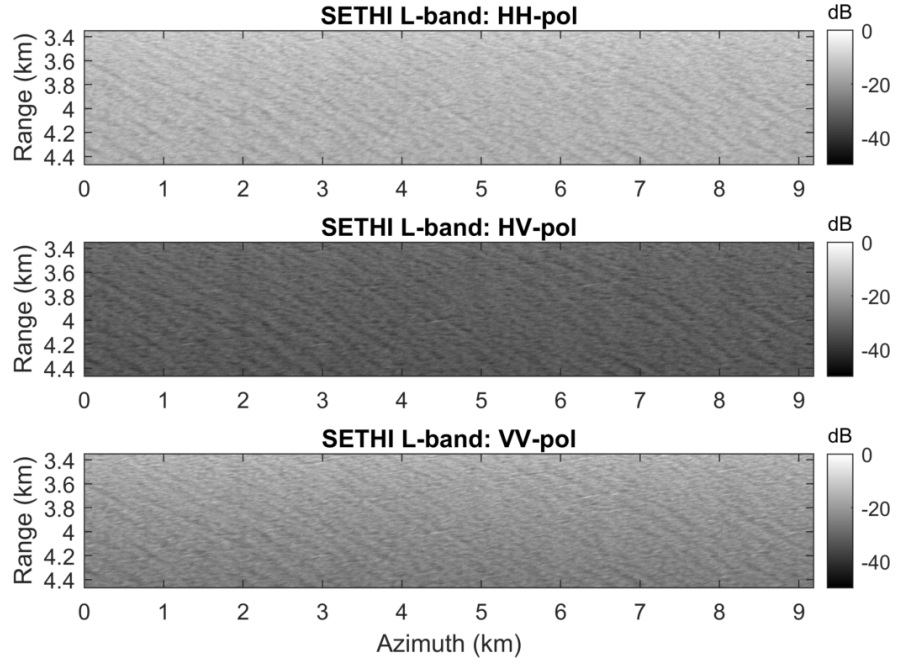

Fig. 4. SETHI L-band SAR data in the upwind direction.

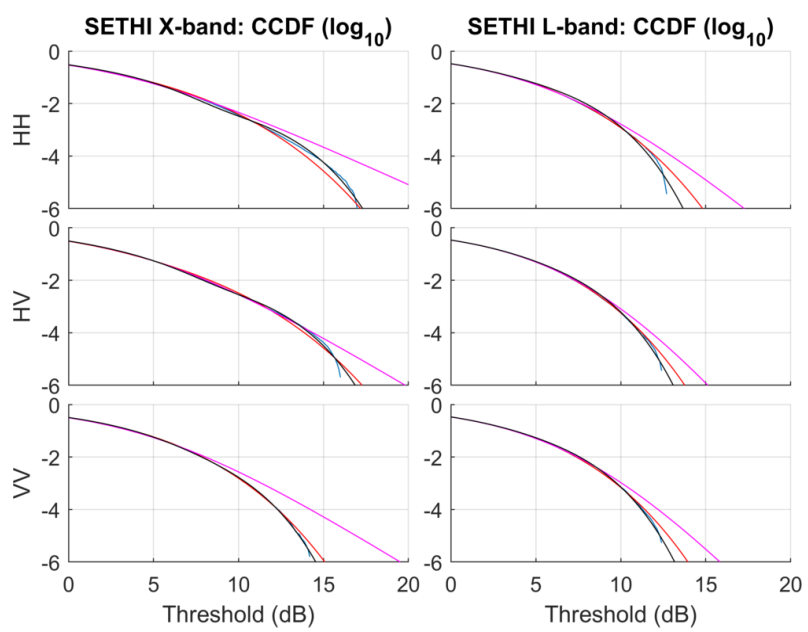

Fig. 5. SETHI SAR CCDF model fits for upwind data and $45^{\circ}$ grazing, X-band (left) and L-band (right). Blue - data, red - K+Rayleigh, magenta Pareto+noise, black - 3MD

TABLE IV. SETHI L-BAND PARAMETER ESTIMATES FOR EXAMPLES IN FIG. 5.

\begin{tabular}{|c|c|c|c|}
\hline & HH & HV & VV \\
\hline CNR $(\mathrm{dB})$ & 41.93 & 36.83 & 45.22 \\
K+Rayleigh shape & 2.06 & 4.05 & 4.32 \\
K+Rayleigh $k_{\mathrm{r}}$-value & 0.27 & 0.21 & 0.12 \\
Pareto shape & 5.30 & 7.86 & 6.75 \\
3MD mode 1 $(a, c)$ & $(0.72,0.28)$ & $(0.70,0.86)$ & $(0.68,0.84)$ \\
3MD mode 2 $(a, c)$ & $(0.28,1.37)$ & $(0.30,1.28)$ & $(0.33,1.28)$ \\
3MD mode 3 $(a, c)$ & - & - & - \\
\hline
\end{tabular}

\section{3MD TEXTURE ANALYSIS}

\section{A. Ingara data set}

In order to further study the modes of the 3MD model, we compare the product of the texture locations $a_{n}$ and the proportion $c_{n}$. Fig. 6 shows this result for each polarisation, over all azimuth angles and $15^{\circ}-45^{\circ}$ grazing. The dashed lines indicate either missing data or regions where no mode was 
TABLE V. X-BAND SETHI SAR MEASUREMENT ERRORS FOR EXAMPLES IN FIG. 5. THRESHOLD ERROR IS MEASURED AT $10^{-4}$.

\begin{tabular}{|c|c|c|c|}
\hline & HH & HV & VV \\
\hline K+Rayleigh BM (dB) & -36.34 & -37.09 & -44.38 \\
Pareto BM (dB) & -33.29 & -42.56 & -33.32 \\
3MD BM (dB) & -35.44 & -41.24 & -41.91 \\
\hline K+Rayleigh threshold error & 0.53 & 0.41 & 0.01 \\
Pareto threshold error & 1.72 & 0.11 & 1.89 \\
3MD threshold error & 0.09 & 0.12 & 0.02 \\
\hline
\end{tabular}

TABLE VI. L-BAND SETHI SAR MEASUREMENT ERRORS FOR EXAMPLES IN FIG. 5. THRESHOLD ERROR IS MEASURED AT $10^{-4}$.

\begin{tabular}{|c|c|c|c|}
\hline & HH & HV & VV \\
\hline K+Rayleigh BM (dB) & -37.54 & -37.91 & -38.48 \\
Pareto BM (dB) & -35.81 & -36.99 & -36.72 \\
3MD BM (dB) & -34.52 & -36.62 & -36.60 \\
\hline K+Rayleigh threshold error & 0.12 & 0.03 & 0.13 \\
Pareto threshold error & 1.07 & 0.56 & 0.91 \\
3MD threshold error & 0.24 & 0.11 & 0.06 \\
\hline
\end{tabular}

required for the model fit. From this result, the $\mathrm{HH}$ polarisation always needs at least 2 modes, with $57 \%$ requiring 3 . For the HV polarisation, $96 \%$ requires at least 2 modes, while $16 \%$ requires 3 . For $\mathrm{VV}, 86 \%$ requires 2 modes, while only $5 \%$ requires 3 .

To highlight common trends between the distributions, the $\mathrm{K}+$ Rayleigh shape estimates are shown in Fig. 7. The first point to highlight is the low grazing angle region in the $\mathrm{HH}$ polarisation, where there are nearly always 3 modes required and an even proportion spread between the first two modes $\left(a_{n} c_{n} \sim 0.5\right)$. This matches where the $\mathrm{K}+$ Rayleigh shape value is low indicating the spikiest clutter. Secondly, there are a number of regions where the $\mathrm{K}+$ Rayleigh shape is high and the 3MD estimates are nearly uni-modal, such as in the HV upwind direction and VV upwind and downwind directions.

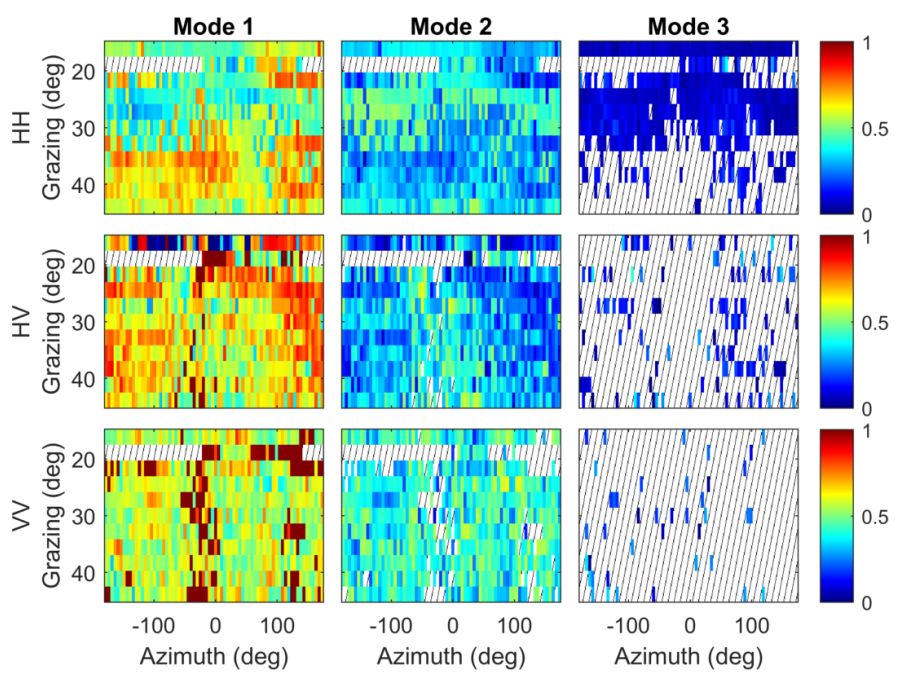

Fig. 6. Ingara data weighted texture modes, $a_{n} c_{n}$ as a function of geometry.

\section{B. SETHI data set}

We now investigate the 3MD parameters for the SETHI data sets, where the data is pooled into blocks of $0.1^{\circ}$ grazing

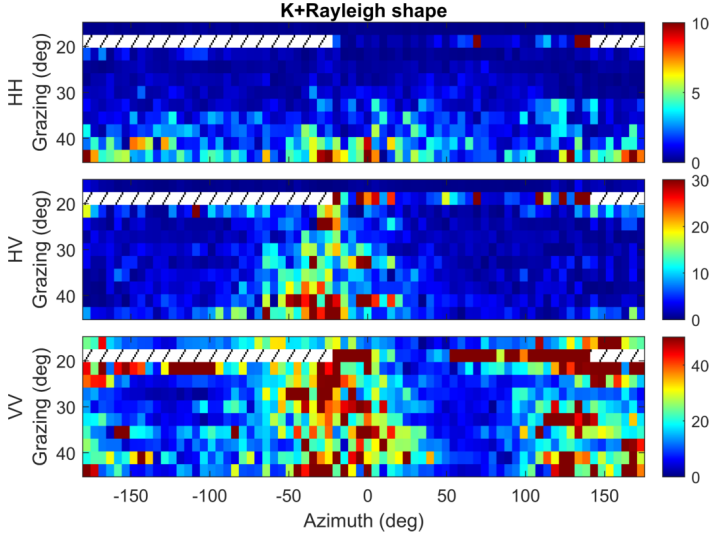

Fig. 7. Ingara data $\mathrm{K}+$ Rayleigh shape values as a function of geometry.

containing approximately $10^{4}-10^{5}$ samples. At X-band, the 3 polarisations always require at least 2 modes, with 3 required in $95 \%$ of $\mathrm{HH}$ and $\mathrm{HV}$ and $62 \%$ in VV. Similarly to the Ingara data analysis, we observe that the third mode is required less for the VV polarisation, while the $\mathrm{HH}$ and $\mathrm{HV}$ polarisations nearly always require 3 modes, which is not the case for the Ingara dataset. At L-band, 2 modes are always needed, with the third required in $11 \%, 4 \%$ and $6 \%$ for the $\mathrm{HH}, \mathrm{HV}$ and VV polarisations respectively. These results are highlighted in Fig. 8 with fits to the SETHI HH data using different numbers of modes. This also confirms the need to take into account more complex backscattering mechanisms at higher frequencies [16], [17].

The weighted texture parameters $\left(a_{n} c_{n}\right)$ are then studied in Figs. 9-10 over the grazing angle range $38^{\circ}-56^{\circ}$. In each case and for each mode, the weighted texture is almost constant with the grazing angle. This is not unexpected as the data lies in the plateau scattering region where there is little variation with grazing angle. We can expect more variation for data collected at either lower or higher grazing angles.
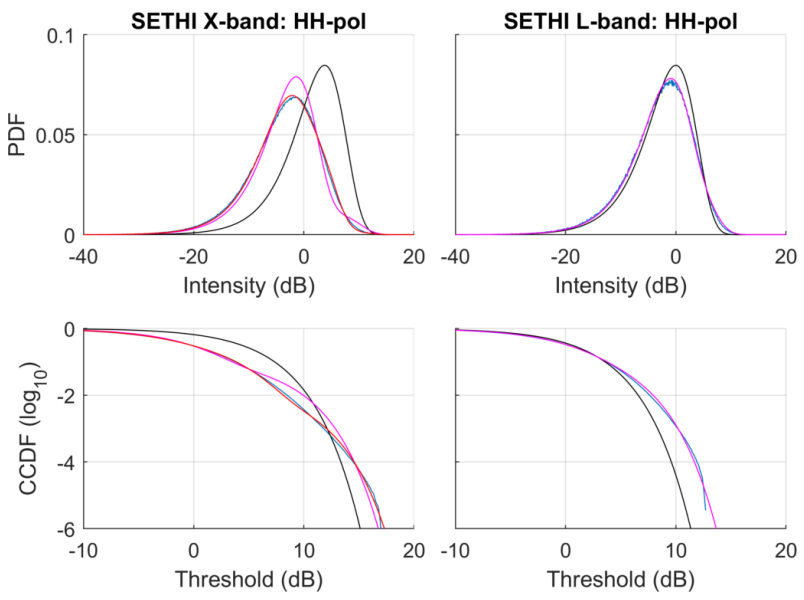

Fig. 8. SETHI SAR data examples of 3MD models fitting. Left panel: $\mathrm{X}$-band, right panel: L-band. Blue: data, black: first mode, magenta: first and second modes, red: all three modes. 


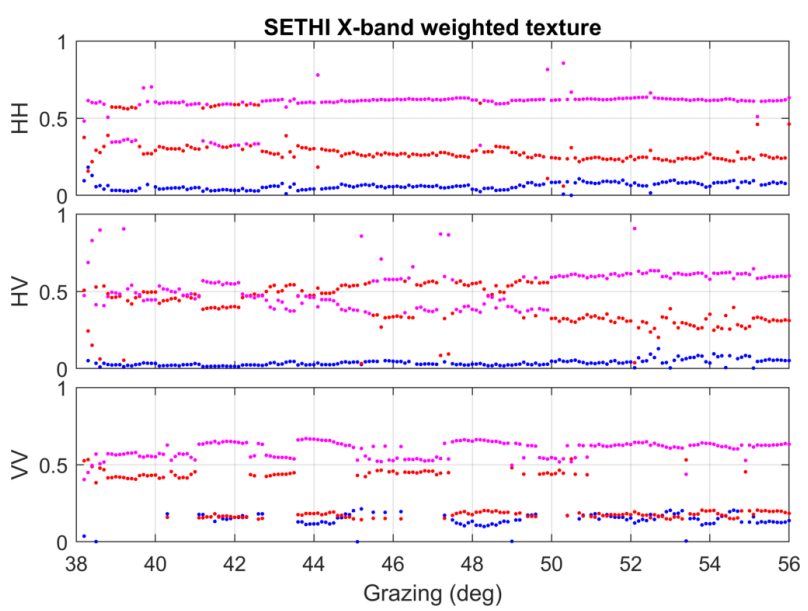

Fig. 9. X-band SETHI SAR data weighted texture modes $\left(a_{n} c_{n}\right)$ variation with grazing angle. Magenta - mode 1 , red - mode 2, blue - mode 3.

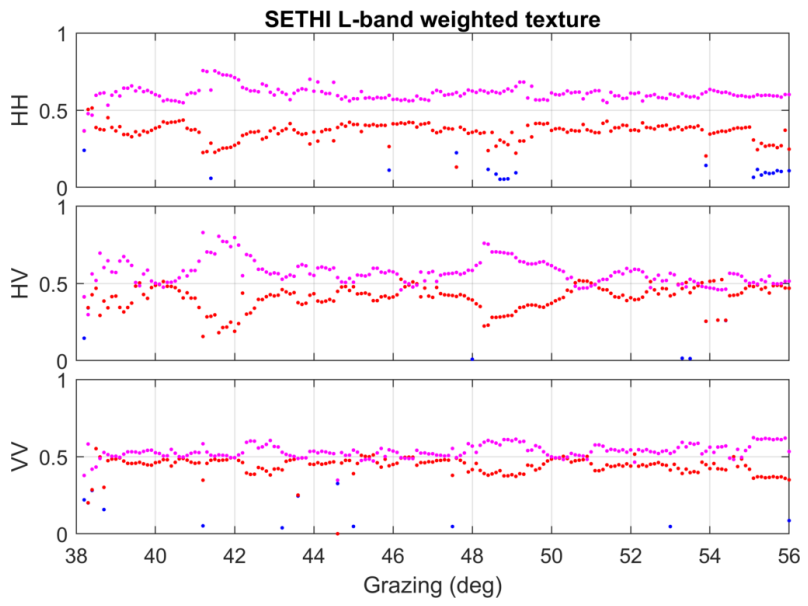

Fig. 10. L-band SETHI SAR data weighted texture modes $\left(a_{n} c_{n}\right)$ variation with grazing angle. Magenta - mode 1 , red - mode 2, blue - mode 3.

\section{CONCLUSION}

In this paper, the 3MD model has been explored using data from the DST Group Ingara radar and the ONERA SETHI SAR. The 3MD model was shown to fit each data set extremely accurately over a wide range of geometries, two different frequency bands and both real aperture and synthetic aperture data. The model texture values were then studied to better understand their relationship to the underlying seaclutter characteristics. For the Ingara data, it was found that $57 \%$ of data from the $\mathrm{HH}$ polarisation requires 3 modes, which was significantly less than the HV and VV polarisations. This is in contrast to the ONERA data which required 3 modes for nearly all fits to the $\mathrm{HH}$ and $\mathrm{HV}$ polarisations. For the Ingara data, the spiky clutter region at low grazing angles in the $\mathrm{HH}$ polarisation nearly always required 3 modes with an even proportion spread between the first two modes $\left(a_{n} c_{n} \sim 0.5\right)$. There were also a number of regions where the $3 \mathrm{MD}$ estimates were nearly all uni-modal, such as in the HV upwind direction and VV upwind and downwind directions. For the ONERA data, it was found that less modes were required for the Lband data implying less complex backscattering mechanisms.

\section{ACKNOWLEDGMENTS}

SETHI SAR data used in this paper have been collected under the NAOMI (New Advanced Observation Method Integration) project, a common research program between TOTAL (the French petroleum company) and ONERA.

\section{REFERENCES}

[1] K. D. Ward, "Compound representation of high resolution sea clutter," Electronic Letters, vol. 17, no. 16, pp. 561-563, 1981.

[2] K. D. Ward, R. J. A. Tough, and S. Watts, Sea Clutter: Scattering, the K-Distribution and Radar Performance, 2nd ed. The Institute of Engineering Technology, 2013.

[3] Y. Dong, "Distribution of X-band high resolution and high grazing angle sea clutter," DSTO, Research Report DSTO-RR-0316, 2006.

[4] A. Balleri, A. Nehorai, and J. Wang, "Maximum likelihood estimation for compound-Gaussian clutter with inverse gamma texture," IEEE Transactions on Aerospace and Electronic Systems, vol. 43, no. 2, pp. 775-779, 2007.

[5] M. Farshchian and F. L. Posner, "The Pareto distribution for low grazing angle and high resolution X-band sea clutter," in IEEE Radar Conference, 2010, pp. 789-793.

[6] G. V. Weinberg, "Assessing Pareto fit to high-resolution high-grazingangle sea clutter," IET Electronic Letters, vol. 47, no. 8, pp. 516-517, 2011

[7] L. Rosenberg and S. Bocquet, "Application of the Pareto plus noise distribution to medium grazing angle sea-clutter," IEEE Journal of Selected Topics in Applied Earth Observations and Remote Sensing, vol. 8, no. 1, pp. 255-261, January 2015.

[8] L. Rosenberg, S. Watts, and S. Bocquet, "Application of the K+Rayleigh distribution to high grazing angle sea-clutter," in International Radar Conference, 2014.

[9] A. Fiche, S. Angelliaume, and L. Rosenberg, "Analysis of X-band SAR sea-clutter distributions at different grazing angles," IEEE Transactions of Geoscience and Remote Sensing, vol. 53, no. 8, pp. 4650-4660, 2015.

[10] C. H. Gierull and I. C. Sikaneta, "Improved SAR vessel detection based on discrete texture," in European SAR Conference, 2016, pp. 523-526.

[11] _ "A compound-plus-noise model for improved vessel detection in non-Gaussian SAR imagery," IEEE Transactions on Geoscience and Remote Sensing, 2017.

[12] S. Bocquet, "Parameter estimation for Pareto and K distributed clutter with noise," IET Radar Sonar and Navigation, vol. 9, no. 1, pp. 104$113,2015$.

[13] D. H. Kil and F. B. Shin, Pattern Recognition and Prediction with Applications to Signal Processing. Springer-Verlag New York, Inc., 1998.

[14] D. J. Crisp, N. J. Stacy, and A. S. Goh, "Ingara medium-high incidence angle polarimetric sea clutter measurements and analysis," DSTO, Technical Report DSTO-TR-1818, February 2006.

[15] S. Angelliaume, X. Ceamanos, F. Viallefont-Robinet, R. Baqué, P. Déliot, and V. Miegebielle, "Hyperspectral and radar airborne imagery over controlled release of oil at sea," Sensors, vol. 17, no. 1772, pp. 1-21, 2017.

[16] E. Makhoul, C. López-Martínez, and A. Broquetas, "Exploiting polarimetric TerraSAR-X data for sea clutter characterization," IEEE Transactions on Geoscience and Remote Sensing, vol. 54, no. 1, pp. 358-372, 2016

[17] V. Kudryavtsev, D. Hauser, G. Caudal, and B. Chapron, "A semiempirical model of the normalized radar cross section of the sea surface, 2. radar modulation transfer function," Journal of Geophysical Research, vol. 108 , no. C3, pp. 2156-2202, 2003 